nodules--peritoneal, glandular, hepatic, and pulmonaryorganisms were demonstrated by Gram's method within the new-formed cells.

GuINEA-PIG Ir.-Inoculated into the peritoneal cavity; killed thirty-seven days later. The nodules in the peritoneum consist of oval and polygonal cells with a large cell body and large spherical and oval nuclei (Fig. 6). Lymphocytes are present throughout the nodule in fair numbers. The arrangement of the cells is similar to that described in Guinea-pig I. The mesenteric glands are occupied by masses of cell growth, which reproduces in the character of the cells and their arrangement the characteristics of the nodules in the peritoneum.

The liver nodules are numerous; the type of the cells composing these nodules and their arrangement are exactly similar to those of the peritoneal nodules. The new cell growth cannot, as in Guinea-pig I, be traced to the-small bile ducts, and its structure is different from that found in Guinea-pig I.

I have recently had the opportunity of examining sections of a peritoneal carcinoma in the hnman subject kindly placed at my disposal by Dr. A. S. Grünbaum, and I find that the structure of this shows a marked resemblance to that of the nodules I have described in these guinea-pigs.

Doc 1 . - Killed forty-two days after intraperitoneal inoculation. Nodules were seen with the naked eye in the omentum, mesenteric glands, liver, and spleen. The omental nodules consist of a parenchyma and a stroma. The parenchyma consists of branched columns of epithelium-like cells irregularly arranged (Fig. 1). They can be traced from the surface endothelium, where their arrangement is in masses, downwards to the deeper parts, where they lie in a dense stroma. The mesenteric glands appear to be infiltrated with new tissue which can be seen with the naked eye. This is similar in structure to the omental nodules in regard to both the character and arrangement of the cells. Nodules in the liver are situated in relation to the portal canals, and between them the liver parenchyma is compressed; they consist of branching columns of cells and of fine tubules in a connectivetissue stroma ; the cells composing the columns and tubules show characters which are identical with those of the epithelium of the bile ducts, but there are appearances also which seem to connect these cells with the parenchyma of the liver lobules, and the exact histogenesis of the nodules is somewhat uncertain. The spleen is studded with nodules; the structure of these is somewhat indistinct, but their general characters resemble those of nodules in the peritoneum.

Dog II.-Killed eighty-two days after inoculation into the mammary gland. A single nodule the size of a large hazelnut was present in the mamma, and there were numerous nodules in the spleen. The mammary nodule was composed of cells with large body and spherical or oval nuclei, supported by a fine connective tissue; lymphocytes were soattered throughout. The large cells are arranged for the most part in parallel columns within the stroma interstices, but the arrangement cannot be said to be regular or to have assumed a definite type. The nodules in the spleen are fibrocellular; they are not marked off from the splenic pulp by any fibrous boundary; the cells are arranged in groups within the connective-tissue stroma, but their histogenesis is uncertain. The stroma is denser than in the mammary nodule, and the nodule on the whole does not reproduce the characters of the latter, although it bears some resemblance to it.

These four examples illustrate the character of the new tissue formation which follows inoculation with the organism; in one animal alone were there nodules in the kidney; these consisted of groups of cells arranged in a distinctly alveolar manner in a tine stroma, and derived by proliferation from the epithelial eells of the renal tubules.

In all the lesions examined the characteristic organisms were demonstrated as intracellular parasites; occasionally intercellular specimens were seen.

Summary of the lnoculation Results.

The organism showed a capacity for initiating active proliferation of endothelial and epithelial cells. The new-formed tissue resulting from this showed a parenchyma and a stroma, and at its edges a progressive growth without encapsulation. In neighbouring glands there was found in some instances a formation of new tissue whose structure resembled that of the primary nodules. The nodules in viscera appeared as a rule to arise from epithelium in loco, and showed a capacity for active growth and infiltration similar to that observed in the nodules arising from endothelium. In one instance, at least, new-formed tissue in the liver reproduced exactly the characters of nodules in peritoneum and mesenteric glands, and, assuming that the observation that it did not arise from cells in loco was correct, must be considered a true visceral metastasis.

Drmonstration of the Organism in Human Carcinoma.

Having found that the organism was demonstrated by Gram's method within the cells of the experimentallyproduced nodules, the application of this method to carcinoma mammae naturally followed.

My observations on this point have up to the present been few in number and relate to three cases only. In these three carcinomata Gram's method shows the presence within the parenchyma cells of bodies which give the same staining reaction (Fig. 3) and have the same general appearance as the parasites in the cells of the experimentally-produced nodules; in the latter they were few in number in most cases, and in the carcinomata also they were not numerous. In the nodules they have been described as occupying vacuoles in the cells outside the nucleus, in the carcinomata they occupy the same position; in both cases they were also seen within nuclei forms which presented the same appearances, but this was an exceptional occurrence.

\section{SDYMARY OF RESEARCHRS}

I. From a considerable proportion (58.3 per cent.) of specimens of carcinoma mammae an organism presenting characteristic features was isolated.

2. This organism presents a life-history in which two cycles were traced - the one a vegetative budding cycle, the other a sporulating cycle.

3. The organism when injected into animals is capable of infecting and inhabiting endothelial and epithelial cells.

4. The organism initiates in endothelium and epithelium a process of proliferation. as a result of which masses of newformed tissue are built up which consist of a parenchyma and a stroma, and grow and extend actively from their centres of origin.

5. This new cell-mass formation may be associated with growth of a similar character in neighbouring glands, and some evidence was also provided that visceral metastasis occurs.

6. Intracellular bodies are demonstrable in carcinomata mammae, which present the same features as the intracellular parasites of the experimentally produced nodules.

7. The evidence derived from these researches points to the conclusion that the organism described is an etiological factor in the morbid process known as carcinoma mammae.

[The expenses of these researches were in part defrayed by a grant from the British Medical Association.]

REFERENCES

1 Proc. Roy. Soc., December 14th, 1899. 2 Thompson-Yutes and Johnston Laboratories Report, vol. v, part $\mathrm{r}$, 1903. ${ }^{3}$ Ibid. \& Proc. Roy. Soe., December I4th, 1899 .

\section{CARCINOMA OF THE COLON AND ITS TREATMENT BY COLECTOMY.*}

[WITH ABSTRACTS OF SEVEN SUCCESSFUL CASES.] By BILTON POLLARD, B.S., F.R.C.S., Surgeon, University College Hospital.

Gentremen,-When your President invited me to read a paper before this Society I was engaged in collecting and following up my cases of colectomy for carcinoma of the colon. I was going to publish a bare record of the cases, but, when your President assured me that it would be difficult to find a subject which, at the present time, would be of more interest to you than that of colectomy for malignant disease of the colon, I determined to recast my paper and give it a somewhat more general character in order to fit it for an address to this Society.

The subject of carcinoma of the colon is, I think, worthy of close attention, for it presents a very hopeful field for surgery if only the cases can be dealt with in an early stage. Given early cases, with the patients in good condition, not only should the immediate results be good. but a large percentage of permanent cures should be possible. Columnar-celled

* A paper read at a meeting of the East Sussex Medico-Chirurgical Society on November ifth, rgo3. 
carcinoma, as it occurs in the intestines, is apparently the least malignant of the carcinomata. Carcinoma of the rectum is well known to persist for a long time without greatly impairing the health, unless it gives rise to obstruction of the bowels, or leads, by local extension, to some grave complication. The same is true of carcinoma of the colon. Its tendency to implicate the neighbouring lymphatic glands, and to reproduce itself in distant organs is slight, and only noticed in late stages of the disease. It has often happened that a carcinoma of the bowel has proved fatal by causing intestinal obstruction, and that no evidence of secondary growths could be detected at the necropsy.

From this it follows that if cases of carcinoma of the colon are diagnosed whilst they are still in an early stage and are palpable disease is removed, there are good grounds for expecting prolonged immunity, even if a permanent cure is too much to be hoped for. But before it is possible to advise a patient to submit to an operation of such apparent gravity as colectomy for a disease which is one of slow progress, and which, at the time the decision has to be taken, may give no indications for an immediately fatal ending, it is necessary to be able to assure him that his chances of recovery from the operation are good. It is not possible to gauge these chances at all accurately at present, more experience is necessary; but it is well known that in comparison with other operations of the first magnitude, the results, both immediate and remote, of colectomy for carcinoma of the colon when unattended with intestinal obstruction, are good. I have myself performed colectomy in seven cases of carcinoma of the colon, and in all the operations were immediately successful, and with the exception of one patient who died from heart disease four years after the operation, they are all alive and well at the present time - at periods ranging from two months to over four years from the time of their operations.

These results would certainly justify us in recommending operation in the early stages of carcinoma of the colon, if only the disease could be diagnosed early. But there is the difficulty. The disease begins so insidiously that in the majority of cases its presence is not suspected until it is already far advanced, and in not a few it is unsuspected until a tumour is accidentally discovered, or until an attack of acute intestinal obstruction occurs.

It cannot be too strongly insisted upon that in cases of chronic illness, however slight, associated with dyspeptic symptoms, a systematic physical examination of the abdomen should be inade. A tumour in connexion either with the stomach or the intestine, or some other organ, may be detected when least suspected.

Let us first of all consider the symptoms which are most commonly found in connexion with carcinoma of the colon. They are just such symptoms as might be expected to be caused by a growth which offered some impediment to the passage of the intestinal contents. Indigestion, in which flatulence is a marked feature, is one of the commonest symptoms. Sometimes patients are troubled with nausea and sickness, but flatulence is an almost constant symptom. Jt is doubtless due to the partial retention and decomposition of intestinal contents. The retention may be more complete and may result in marked constipation with the accumulation of scybalous masses of faeces on the proximal side of the growth. The seybala cause irritation of the mucous membrane and, by inducing effusion of fluid into the bowel, set up a sort of spurious diarrhoea, This symptom has been a characteristic one in several of the cases which have come under my notice. Alternating constipation and diarrhoea is, as is well known, a frequent symptom of chronic obstruction of the large intestine. In most cases pain occurs sooner or later. It may be slight and not very distinctly localized, or it may be constantly referred to some particular spot. It may, at first, be no more than a feeling of discomfort, which may be relieved or aggravated by some particular movement. But in many cases, without any definite obstruction occurring, the pain is very severe. It comes on in attacks and is evidently due to intestinal colic. This symptom was very marked in my third and fifth cases. In Case III it was associated with much flatulence. The growth was situated in the splenic flexure, and the patient described the attacks of pain as beginning in the right iliac fossa and travelling along the course of the colon to the left hypochondrium. The attacks of pain got more and more severe until he felt a gurgling and the passage of flatus down the left loin, when he obtained relief. - In Case $\nabla$ the pain was greatly aggravated by meals, particularly of solid food ; and in consequence of this symptom the patient had, for some months before his operation, restricted himself to liquid food. It is not surprising that patients who have been troubled with these symptoms should have lost flesh; and in all my cases lose of flesh had been a marked feature, but they had all reached a fairly advanced stage when they came under my notice.

When most of the symptoms already mentioned occur together, we get a fairly characteristic clinical picture, and when there is added to them another symptom, the evidence of a growth in the bowel is nearly complete. The symptom I refer to is haemorrhage from the bowel. The bleeding may be only sufficient to cause melaena, or it may be profuse enough for unaltered blood to be passed from the bowel. In two of my cases-Cases III and v-a quantity of bright blood was passed per rectum.

In the presence of some or all of these symptoms a careful physical examination should be made, and very probably an abdominal tumour will be readily detected. If the surgeon is in doubt, it is much wiser to insist on the necessity of making an examination under an anaesthetic, with relaxed abdominal walls, than to run the risk of missing the tumour.

In some cases the symptoms are slight and do not trouble the patients much; and, indeed, the tumour itself may be the first thing to definitely arrest their attention. This was so in my second, sixth, and seventh cases.

So long as there is no obstruction of the bowels, with consequent abdominal distension, the detection of a large tumour of the colon is generally easy. Sometimes, however, the growth is quite small although it constricts the bowel considerably. A growth of this kind would be very difficult, and perhaps impossible, to feel, especially if it occupied the splenic flexure and was hidden in the left hypochondrium, or if it occupied the sigmoid flexure and, owing to the presence of a long meso-colon, hung down into the pelvis.

In an ordinary case there is a tumour to be felt somewhere in the course of the colon by abdominal palpation. The seven cases which I am at present chiefly considering were located as follows-two in the ascending colon, two about the splenic flexure, one in the descending colon, one about the middle of the sigmoid flexure, and one at about the junction of the latter with the rectum. I have also met with cases of carcinoma of the caecum and of the hepatic flexure of the colon, but I do not recollect a case in which the transverse colon was primarily involved at a distance from the hepatic and splenic flexures. Such cases have been recorded, however, and I have met with a case in which the middle of the transverse colon was invelved by extension of a carcinoma which was primarily situated in the small intestine-at the beginning of the jejunum. It follows, therefore, that the tumours of the colon are generally situated in the lateral parts of the abdomen-in the hypochondriac, lumbar, and iliac regions.

In the early stages, before the growths have become adherent to the abdominal wall, they are very movable, and then, in the absence of localizing symptoms, the question of diagnogis from movable kidney has often arisen. This question actually did arise in two of my cases. In Case III the growth occupied the lower part of the left hypochondriac and the upper part of the lumbar regions. It was so movable that it could be displaced upwards entirely beneath the ribs on the left side as well as quite over to the opposite side of the abdomen. This tumour was thought by another surgeon to be a movable kidney, and the symptoms and.signs-flatulence, severe attacks of colicky pains and haemorrhage from the bowel-were attributed to other causes. Now, in my experience, it has generally proved to be true, when there were well-marked symptoms and a well-marked physical condition which could quite reasonably be the cause of these symptoms, that the physical condition was really the cause of the symptoms. Relying on this general principle, I was strongly of opinion that in this case, although the patient was only 29 years of age, we had to do with a tumour of the large intestine. This view gained support from the superficial position of the tumour and the irregularity of its outline, and especially from the fact that a swelling, which I took to be the normal kidney, could be felt behind the tumour, and unconnected with it, in the proper position for the kidney. In another case-No. VII-the possibility of the tumour being a displaced kidney was raised with more justification, for the tumour had somewhat the shape of the kidney, and had a smooth surface, and the patient complained of frequency of. micturition, and made no other complaint until he was questioned. He then said that he had been troubled with diar- 
rhoea on and off during the last two months, and that he had not passed a formed stool during the last three weeks, that he had been subject to slight attacks of pain in his left side, and that he had latterly been losing flesh. In this case the tumour had not much mobility. It could be moved a little lateraliy and a little downwards, but hardly at all upwards. Now, in my experience, a displaced kidney can always be returned by manual pressure to the normal position of a kidney, and I am inclined to rely absolutely on this sign.

It is, of course, obviously possible for a displaced kidney to get fixed by perinephritis, but then there would be evidences of kidney disease in the state of the urine. I have drawn attention to the fact that carcinoma of the colon gives rise, to begin with, to a movable tumour, and that it is owing to secondary adhesions that the mobility is lost later on. This was so in Cases IV and v, and to a less extent in Case vII, in all of which the bowel had become adherent to the parietal peritoneum.

Carcinoma of the colon may become adherent to another organ, and thus acquire the physical signs of that other organ. This possibility is well illustrated by my second case, in which a carcinomatous tumour of the splenic flexure of the colon had extended into the left kidney, and the two together formed a tumour which I thought was a renal tumour. Its physical signs were exactly like those of a renal tumour, and although there were no urinary signs of renal disease, the symptoms were not inconsistent with a renal source.

A carcinoma situated at about the hepatic flexure of the colon might, so far as its physical signs were concerned, resemble an enlarged gall bladder or a growth of the pyloric portion of the stomach. I had such a case a few months ago, but it proved to be inoperable. The patient had been ailing and losing flesh for two years. He complained of discomfort in the abdomen just below the ribs on the right side. Under an anaesthetic a lump could be felt there. The only localizing symptoms were occasional sickness and diarrhoea. The vomit had only consisted of partially digested food; it had never been very voluminous, and had never contained blood. The tumour was a big one, and, if it had been situated in the pyloric portion of the stomach, I should have expected more evidence of pyloric obstruction. In these circumstances the tumour was correctly diagnosed as carcinoma of the hepatic flexure of the colon.

It must not be forgotten that a tumour of the splenic flexure or the neighbouring part of the transverse colon may invade the stomach, and that then stomach symptoms may overhadow those due to the original disease in the colon.

There were special difficulties in diagnosis in Case $\mathrm{v}$ owing to the position of the growth. The symptoms, however, were of themselves almost conclusive. Attacks of griping pains had been of daily occurrence for nine months before the operation they were much worse after food, and particularly after solid food. Loss of appetite and loss of flesh had been marked for seven months. Aperients had been required regularly during the previous half-year and on many occasions the motions had contained blood. Malignant disease of the large intestinewas of course suspected to be the cause of these symptoms, but, at first, no tumour could be detected either by abdominal or rectal examination. Another surgeon had examined the patient with the assistance of an anaesthetic and had failed to detect the growth. The patient was brought to me on the advice of Dr. Sidney Martin by Dr. Dill Russell, of Fortis Green, who had been able to throw much light on the case. He had found a piece of new growth in the motion and he had succeeded in feeling the tumour by rectal examination. When I saw the patient first, neither Dr. Russell nor I could feel the tumour, but I was satisfied that it was there, and I arranged to operate. Dr. Russell, however, felt it again a few days later, and on the day before the operation I succeeded without an anaesthetic in feeling the tumour quite distinctly by rectal examination. The mucous membrane of the rectum was normal, and the growth, which could be felt to be outside the rectum, was evidently connected with the bowel higher up. At the operation it was found to be situated at the junction of the sigmoid flexure with the rectum. In this case the evidence pointing to the presence of a tumour of the colon was very strong, and I determined to operate although I could not at that time feel the tumour. But I think it would be right to do an exploratory operation in cases in which the symptoms were less definite than they were in this case, provided that they were characteristic and persistent; for it must not be forgotten that some carcinomatous growths of the colon, although they cause a good deal of constriction of its lumen, are so small that they cannot be felt through the abdominal wall.

Now I come to the subject of treatment, and, in regard to that, there are two classes of cases to be considered-those in which there is acute obstruction of the bowels and those in which there is no material obstruction. It is mainly with the latter class of cases that I am at present concerned, but with regard to the former a few words will not be out of place, particularly as one of my cases belonged to that class.

In cases of acute obstruction due to carcinoma of the colon it will rarely be possible to feel the growth, and the exact site of the obstruction will be incapable of determination. No reliance can be placed on statistics of relative frequency. It would, undoubtedly, be a mistake to do typhlotomy with the idea that the obstruction would certainly be relieved by opening the caecum. A better procedure would be to explore the abdomen through a median incision below the umbilicus, as I did in Case I, and possibly the surgeon may be fortunate enough to find, as I was in that case, that the growth is situated in a portion of bowel sufficiently mobile to allow of its being brought up to the surface. If the piece of bowel containing the tumour can be withdrawn from the abdomen, the obstruction may be relieved by opening the bowel on the proximal side of the growth and tying in a Paul's tube. Later on the piece of bowel containing the growth may be excised, and still later the continuity of the intestinal canal may be re-established. It the growth cannot be readily brought out of the wound I think it best not to meddle with it at all at that time, but to relieve the obstruction by making a temporary artificial anus higher up, and at a later date, on the subsidence of all acute symptoms, to remove the growth through an incision made directly over it.

And now let me turn to the consideration of the other class of cases: those in which there is no material obstruction of the bowels, but in which the symptoms and physical signs have led to the diagnosis of carcinoma of the colon-cases in which a tumour can be felt. As a general rule the incision should be made directly over the tumour, and it should be made long enough to allow of the necessary manipulations being readily carried out. Want of room uselessly hampers manipulation and adds to the risks of the operation in severa ways. If the growth is at the upper end of the rectum and cannot be felt through the abdominal wall, as in Case $v$, the incision may be most conveniently made in the lower part of the left linea semilunaris.

Having opened the abdomen, the surgeon must, first of all decide whether the operation of colectomy is justifiable, and, if so, whether it seems possible to carry it through successfully. He must make a thorough examination of the tumour and its connexions, and search for secondary growths both in the lymphatic glands and in the liver. If there are secondary growths in the liver, or if the lymphatic glands are the seats of undoubted and widespread secondary growths, it would not be justifiable to expose the patient to the risks of colectomy. But in this connexion it should be borne in mind that mere enlargement of the lymphatic glands does not of necessity imply carcinomatous infection. Both clinical and pathological experience has shown that the glands may be considerably enlarged without their being carcinomatous. In Cases IV and vi the lymphatic glands were much enlarged, but not carcinomatous, I think. It is now over two years since Case Iv was operated upon, and there is no evidence of recurrence. It is only six months since Case vi was operated upon and so the clinical evidence is not so conclusive, but in that case I re moved a few of the largest glands, and the Surgical Registrar at University College Hospital examined them carefully, but failed to detect any evidence of carcinomatous infection. Mere enlargement of lymphatic glands then should not, I think, contraindicate colectomy.

But before commencing to separate the bowel the surgeon should carefully examine the relations of the growth and satisfy himself that it is removable. It may have involved the abdominal wall too extensively for removal or it may have invaded some neighbouring organ of importance which it is not feasible to remove, or, on the other hand, which could be removed, if only the patient. were strong enough to bear the operation. If the growth had involved a coil of jejunum or ileum its removal would still be possible if the patient's condition was good enough to warrant the surgeon's subjecting him to a double enterectomy. I have removed the sigmoid flexure of the colon together with three feet of small intestine myself in a case which at the time of the operation I thought was one of carcinoma of the sigmoid flexure to which some coils of ileum had become firmly connected by 
inflammatory adhesions. The patient made a normal recovery, and the case has been already reported. It is not, however, included in this series of cases, abstracts of which are in your hands, because subsequent examination showed the tumour and the adhesions to be of a simple inflammatory nature. I have had two other cases in which both the large and small intestine were involved by undoubted carcinomatous tumours; but in neither of these cases was complete removal of the diseased structures possible.

In one the primary growth was in the hepatic flexure of the colon, and it had spread back into the second part of the duodenum and had implicated the anterior and inner parts of its circumference. Had it been possible to perform the double enterorrhaphy satisfactorily there would still have been the common bile duct and the pancreatic duct to deal with, and they would have presented insuperable difficulties, I think. In the other case the tumour involved the jejunum just at its commencement and the colon in the transverse portion. The latter could have been dealt with readily enough, but in order to remove the growth in the jejunum it would have been necessary to divide the bowel close to the termination of the third part of the duodenum. Owing to the immobility of this portion of the duodenum the reunion of the divided small intestine seemed impossible. And further, it seemed likely that removal of the growth would have involved injury to the superior mesenteric artery-a necessarily fatal accident. In this case a short-circuiting operation was done and it afforded great relief to the patient.

Carcinoma of the transverse colon is also liable to invade the stomach, but that is not a complication which would present insuperable difficulties to the surgeon unless the extent of stomach involved was too great for removal. Solid viscera may be implicated also, as happened in my second case, in which the growth, primarily situated in the splenic flexure of the colon, had extended into the kidney and the tail of the pancreas, and necessitated the removal of the kidney and a portion of the pancreas, together with the splenic flexure and neighbouring parts of the colon.

In those cases in which the radical operation of colectomy is impossible, owing to the infiltration of the growth into irremovable structures, or in which it is not justifiable to expose the patient to the risks of colectomy, because of the presence of secondary growths incapable of removal, relief may be afforded by the less serious operation of shortcircuiting.

Before commencing to isolate the piece of bowel containing the growth, the surgeon should try to determine what length of bowel will need removal. Now this will primarily depend on the extent of bowel involved in the growth, but it will also depend upon what is called for in order to make a satisfactory union of the divided ends. In cases of carcinoma of the ascending colon, the surgeon will have to determine whether the caecum will have to be removed or not. This decision will depend on the extent of healthy bowel which intervenes between the growth and the caecum. In Case iv it was un necessary to remove the caecum because, after division of the colon at a sufficient distance from the growth, there was still enough bowel above the ilio-caecal valve to suffice for its union with the distal segment. In Case vi it was not possible to preserve the caecum, because the mere removal of the growth would have necessitated division of the colon just above the ileo-caecal valve.

Again, in order to perform the operation with the least risk of septic infection it is necessary that both the divided ends should be sufficiently mobile to allow of their being manipulated outside the abdomen. In both the cases just referred to it was necessary to remove the whole of the upper part of the ascending colon because it was involved in the growth. Consequently, the distal section lay in the transverse colon, which, owing to its long mesocolon, was mobile enough to be brought into contact with the proximal end But sometimes the needs of the enterorrhaphy call for the removal of more bowel than would have been required if the mere eradication of the growth had been all that needed consideration. This point is illustrated by Case vII, in which the growth occupied the lower end of the descending colon and in which adequately wide removal of the growth could have been effected by division of the bowel below the splenic flexure. But section at that spot would not have provided two segments of sufficient mobility to allow of their being brought together without causing great tension. and still less to permit of their being brought outside the abdomen during the performance of the operation. As is well known. the splenic flexure of the colon is held firmly up by the pleurocolic fold and the other peritoneal folds, and, in the case under consideration, it was only after these folds, together with the blood vessels contained in them, had been divided and the transverse colon reached that a sufficiently mobile piece : of gut was ob'ained. At the lower end, also, it was necessary, with the same object, to divide the bowel well within the sigmoid flexure. Cases of colectomy in the course of the ascending and descending portions of the colon have been recorded in which, owing to want of attention to this point, sloughing of the bowel, which was attributed to strain on the mesocolon, had occurred. And, again, in considering this same question - the length of bowel which should be removed-it is all-important to consider the vascular supply of the pieces of bowel to be united. If there is any suspicion of the adequacy of the vascular supply, a little more of the bowel should be removed. For instance, in Case vit, in separating the attachments of the caecum, it appeared that the blood supply of the lower end of the ileum had been interfered with; and so, in order to avoid any risk of sloughing, 5 in. of the ileum were removed as well.

Having decided to proceed with the colectomy, and having settled upon the length of bowel to be removed, the surgeon should carefully isolate the field of operation from the rest of the abdomen by packing in large folds of sterile gauze. He may then begin to free the bowel. The growth, together with the whole pier.e of bowel to be removed, should be loosened from all its attachments by ligaturing and dividing the mesocolon and other peritoneal folds connected with it. In doing this the greatest care must be taken of important structures in relation with it, such as the ureter and the renal vessels. Of course, if those or other removable structures were involved in the growth they would have to be sacrificed. In Case II the growth had invaded the kidney, and the renal pelvis; and in consequence the kidney was taken away along.with the tumour. The same treatment would in all probability be required if the ureter was involved.

In separating the parts to be removed it is necessary to be constantly on the look-out in order to avoid injuring blood vessels supplying other tissues, and particularly the stomach and portion of intestine which are not going to be removed. The piece of bowel being separated, it should be withdrawn from the abdomen and emptied by passing it through the fingers. It should then be clamped two or three inches beyond the places at which the bowel is to bedivided, and another pair of clamps should be put on the bowel to be removed close to the points of section. After trying various kinds of elamps I have come to the conclusion that there is nothing better for the purpose than Doyen's forceps.

At this stage the gauze packing, which has been used to isolate the field of the operation from the rest of the abdomen, should be changed, and additional strips of gauze should be packed into the wound beneath the separated loop of bowel, and particularly about the lines of section, in order to mop up anything which escapes from the bowel when it is cut across. The section of the gut must be made close up to the unseparated mesentery or mesocolon, and it is well to make the sections rather obliquely, more bowel being removed on the side opposite the mesenteric attachment, so that they may lie within an area of as good vascular supply as possible. In removing the piece of bowel the greatest care should be taken to avoid contamination of anything remaining in the field of operation. If at this time, or at any time, any of the gauze packing gets contaminated, it should be removed at once and replaced by fresh. The interior of the pieces of bowel about to be stitched together is a constant source of contamination of the fingers, and, through them, of the exterior of the bowel, and even of the abdominal wall. In order to reduce this risk to a minimum the open ends of bowel beyond the clamps should be thoroughly wiped out with little swabs of diy gauze held on forceps, and finally the mucous membrane of these portions of the bowel should, so far as this is possible, be disinfected by mopping it over several times with swabs of gauze moistened with some antiseptic-such, for instance, as $I$ in $\mathbf{r}, 000$ solution of perchloride of mercury.

Everything is now ready for the union of the bowel. In my opinion simple stitching is preferable to any form of mechanical contrivance with which I am acquainted, and I have used it exclusively during the last four years in all my operations on the stomach and intestines. In Case II of this series I used a Murphy button, but not because at that time I preferred it to simple suture, but because the operation had been a severe one and had caused so much shock that $\mathrm{i}^{\mathrm{t}}$ was thou rht advisable to complete it as quickly as pos- 


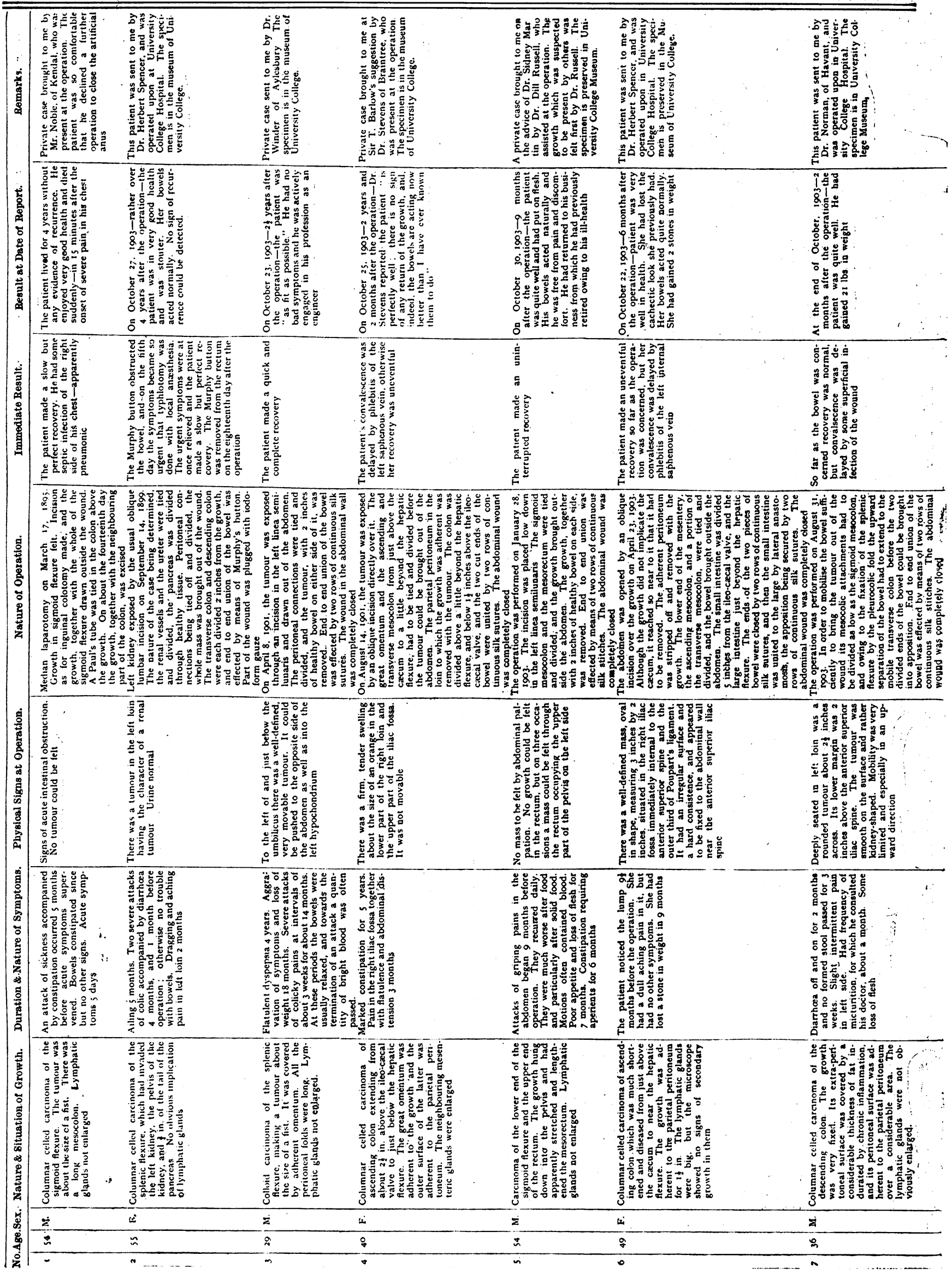


sible. I used the largest size button, and the patient finally did well and she is still alive and well, but the button was very nearly the cause of her death. In the small intestine the Murphy button may possibly be used safely, but in the large bowel its use is attended with an additional risk, which is well illustrated by this case. The lumen of the bowel evidently got blocked with faeces. No flatus was passed per rectum after the third day after the operation, and on the fifth day I found the patient in a very collapsed state, with a very rapid and feeble pulse; faecal vomiting, too, had come on, and there was great abdominal distension. Without delay and under local anaesthesia I made a little opening into the caecum and tied in a small glass tube and gave vent to a huge quantity of gas. The alarming symptoms then quickly subsided. But if simple stitching is adopted there is still ample choice of methods. It is at present a vexed question whether end-to-end union or lateral union is the better. It is possible to find arguments in favour of each method, and these were well stated by $\mathrm{Mr}$. Makins at the last meeting of the British Medical Association, but experience will alone decide which plan is really the better one. Personally, I must confess to a preference for end-to-end union whenever it is possible. I have only had occasion to perform the operation of enterorrhaphy eight times in cases of chronic intestinal obstruction. In seven of the cases end-to-end union was established, and in the remaining case lateral union was effected. All the cases cecovered, and they are all still alive, and so no comparative conclusion can be drawn from the results of my experience. In the case in which I reunited the bowel by lateral anastomosis I selected this method on account of the inequality of the lumina of the two pieces of bowel. It was in Case vi of this series in which the ileum had to be united to the transverse colon.

In this paper I shall only refer to the way in which I perform end-to-end union, because I think that method is the one which is most generally applicable to enterorrhaphy after resection of portions of large intestine for carcinoma. The description of the operation has been already carried to the point where the two ends are ready to be united. The pieces of bowel are first of all linked together by an interrupted stitch at the mesocolic attachment, and then the first row of continuous stitches are inserted from the mucous surface through all the coats of the bowel. I commence the suture at a point well removed from the .attachment of the mesocolon-at a place where the bowel has a good peritoneal covering. An experienred assistant is a great help at this stage of the operation, for he is practised in holding the two segments of bowel in apposition, first with his fingers and then with forceps, in such a way that the stitches can be inserted from the mucous surface for fully fivesixths of the circumference. The final sixth of the circumference is obliged to be stitched from the outside-a method which is both slower and less efficient than stitching from the mucous surface. Owing to foresight in commencing the continuous suture at the spot mentioned the weak part of the first row of stitches-the final sixth--will fall at a part of the bowel where the peritoneal covering is compiete, and therefore at a part most favourably situated for being securely controlled by the second row of continuous sutures, which will be inserted from the peritoneal surface according to Lembert's method, and will penetrate all the coats except the mucous. The first row of sutures serves a double purpose. First of all it controls bleeding, and, with this object, the loops of the suture should be drawn tight. If each loop is tightened before the next is inserted there will be no tendency to constrict the bowel by the running together of the loops in the way the loops of a pursestring suture run together. In the second place, it serves as a first line of defence against leakage, and in order that it may fulfil this function as thoroughly as possible great care should be taken to bring peritoneum to peritoneum, and, where there is no peritoneum, muscular coat to muscular coat. Accurate appposition can be assured so long as the stitches can be inserted from the mucous surface ; but just at the last, when the sutures have to be put in from the peritoneal surface, it is, I think, impossible to prevent a little mucous membrane showing outside here and there between the loops of the suture. The most difficult part to stitch satisfactorily is that in the neighbourhood of the mesocolic attachment, and it is in order to reduce this difficulty to a minimum that the suture is planned in such a way that the mesocolic portion comes where the stitches can beeasily inserted from the mucous surface. The outside suture-the continuous Lembert suture-is the chief defence against leakage.
This row of sutures should get as secure a hold of the coats of the bowel as is possible without penetrating the lumen. It should involve the serous, muscular, and submucous coats, but on no account should it implicate the mucous coat. The surgeon's muscular sense soon learns to gauge the amount of resistance offered to the needle. This is distinctly greater when the needle, punctures the mucous coat than when it avoids it. If the surgeon is in doubt whether he has passed the needle too deeply or not he had better. withdraw it and reinsert it more superficially. The clamps may be removed after the first line of sutures has been imserted. The gap left in the mesocolon should be closed, if possible, by suturing the two sides together, but if this cannot be done, the cut edges of the mesocolon should be stitched down to some surface, so as to close any opening through which small intestine might pass. The bowel in the neighbourhood of the suture line should be dabbed with a i to 1,000 solution of perchloride of mercury and then washed over with sterile salt solution. After the removal of the gauze packing the sutured bowel may be returned to the abdomen. Finally, the wound in the abdominal wall may be completely closed. That is the course I have adopted in all my cases except one. The exception was Case II of this series. The oozing surface left by the removal of the kidney and a portion of the pancreas was so large that it seemed imperative to provide some drainage. This was supplied by a piece of gauze. In an ordinary case there is not likely to be much oozing, and consequently there is no need for drainage.

I have entered with what may be thought to be rather tedious detail into the method of operating which I employed in these cases, and my excuse is that I consider success in the field of intestinal surgery can only be obtained by attention to the minutest details of technique.

The treatment of the cases after operation was conducted on essentially the same lines in all. A rectal injection of normal saline solution was given a few hours after the operation in order to relieve thirst Four hours later rectal feeding was begun. The feeds werc given every four hours, and consisted generally of a suppository of beef peptone alternated with a nutrient enema containing $2 \mathrm{oz}$. of Gerrard's peptones, an ounce of water, and the yolk of an egg. The rectal feeding was continued for four to seven days, according to the tolerance of the rectum. By mouth sips of hot water were allowed from the first, and on the day after the operation some nourishment was given by mouth hourly or every two hours. This consisted, as a rule, of one or other of the following: Half an ounce of albumen water, two teaspooniuls of liquid peptones diluted with an equal quantity of water, two teaspoonfuls of peptonized milk with an equal quantity of barley-water, or a teaspoonful of raw meat juice in half an ounce of water. The amounts were gradually increased, so that by the end of a week $2 \mathrm{oz}$. were given every hour. Food was now only given every second or third hour, and the quantity given at a time was correspondingly increased. The patient was at this time allowed to masticate the lean part of an underdone chop and to swallow the juice, but not the solid. A little bread and butter was given once a day, and some Benger's food two or three times a day. By the end of a fortnight after the operation the patient was usually taking light solid food.

In the early days after the operation, whilst the nutrient enemata were used. the rectum was washed out every morning and then half a pint of normal saline solution was injected into the bowel and left in. A rectal tube was frequently passed in order to give vent to flatus, and thus to prevent, as far as possible, strain on the line of sutures. The patients were instructed not to strain in their endeavours to pass flatus, but to ask the nurse to introduce the rectal tube whenever they felt the presence of flatus in the bowel. As a rule the bowels acted as the result of an oil enema, followed by a simple enema on the tenth to fourteenth day after the operation. If necessary a dose of castor oil was given about a fortnight after the operation.

I now come to a consideration of the results, and it is very satisfactory to be able to report that all my cases made good recoveries. Convalescence was delayed, however, in some of the cases by the occurrence of phlebitis of the superficial veins of the leg. It is curious how often phlebitis complicates operations on the bowels. It occurred in three of these cases - the fourth, fifth, and sixth, and in each case it was on the side opposite to that on which the operation had been performed that the phlebitis developed. I have noticed the same complication in cases of appendectomy. I do not see how to explain this complication. Doubtless one factor is 
some slight septic contamination of the blood, owing to the operation involving a naturally septic region like the bowel; but there must be some other factors, the nature of which is not obvious. The only other complication was, in one case, septic infection of the abdominal wound, which led to separation of some of the buried silk sutures. The deep part of the wound was not involved, and the bowel healed normally.

Immediately successful results are very gratifying to the surgeon as showing the perfection of his operative technique, but, unless the remote results were good also, there would not be much encouragement to perform operations which are attended by so many grave risks. I have already stated that with the exception of the first patient on the list, who lived in complete comfort for four years and then died suddenly from heart disease, all the patients are alive and in good health at the present time, and they have all put on flesh. All the patients have been examined recently, and no evidences of recurrence can be detected either from the presence of symptoms or by physical examination of the abdomen. In three cases sufficient time has elapsed to give good grounds for hoping that the cure in them will be permanent. One case was operated upon over four years ago, another two and a-half years ago, and the third two years and two months ago. The remaining three cases were operated upon too recently-nine, six, and two months ago-to warrant any prediction regarding ultimate prognosis.

There is but little more for me to say. It is unnecessary for me to describe the parts removed, for the specimens are here, ready for your inspection; and, as they will be preserved in the museum of University College, they will be open to the inspection of any one else who is interested in the subject. I need only state that in five cases the growths were columnar-celled carcinomata, and that in one, Case III. the growth, which was a very large one, was a colloid carcinoma.

\section{RADIUM BROMIDE :}

NOTES ON SOME CASES IN WHICH IT HAS BEEN TRIED.

By JAS. MACKENZIE DAVIDSON, M.B., C.M. Medical Officer in charge of Roentgen-ray Department, Royal London Ophthalmic Hospital, and Charing Cross Hospital.

IT seemed highly probable that the radiations from radium bromide, considering their nature and properties, would prove serviceable in the treatment of cases which were benefited by $x$ and ultra-violet rays (Finsen treatment). In the early part of May, 1903, I obtained 5 milligrams of radium bromide in a sealed glass tube.* The exact strength of the radium bromide I use I am unable to state in figures. $\mathbf{M r}$. Soddy, who has done such excellent work on radium, kindly examined it for me, and it is said to be of the finest quality.

It may be well to preface the notes of cases in which I have used it by stating briefly some of the physical properties of this remarkable substance which concern us. In the solid state it gives off three kinds of rays;

I. Alpha Rays.-These are minute material particles propelled at an enormous velocity, and which, if they impinge on a suitable fluorescent screen, cause brilliant scintillations. This was first noticed by Sir William Crookes, who has called the little instrument devised to show the phenomena the "spintharoscope." The glass tube in which the radium is enclosed entirely cuts off all these $a$ rays, so that their action in the cases presently to be described was nil.

2. Beta Rays. - These rays are similar to the cathodal rays inside a Crookes's tube, but have a much higher velocity and consequently have the power of penetrating the glass tube and our tissues to some considerable depth. It seems pretty certain that it is these rays which cause the burning and destructive action on the skin.

3. Gamma Rays.-These resemble in some respects $x$ rays of extraordinary penetrating power, somewhat similar to the rays produced by an extremely high Crookes's tube. The glow on a barium platino-cyanide screen can be seen from one of my 5-mg. tubes through half an inch of copper.

The many other extraordinary properties of radium, such as its power of producing heat and its emanations when in

* Sir William Crookes gave me the address of a firm in Germany likely to supply what I wanted, and this address I handed to Mr. Martindale, o, New Cavendish Street, W., who obtained it for $m$,
I have from time to time obtained further supplies. solution, etc., have no bearing upon the method employed in the following cases, and so nothing need be said about them here. The action upon a photographic plate is rapid and intense. If the salt be shaken down to one end of the tube, and this then used to write upon a photographic plate enclosed in the usual opaque envelopes for $x$-ray tork it will be found on development to show the writing extremely black.

\section{CASE I.-Rodent Ulcer.}

Mrs. F., aged 69, was sent to me at the $x$-ray department, Charing Cross Hospital, by my colleague, Dr. Macleod, for $x$-ray treatment.

There was a typical rodent ulcer on the right side of the nose, the uper border being close to the inner canthus. It was $x$ in. in length by 3 in in breadth, and had resisted both Finsen and $x$-ray treatment. I was glad to have this opportunity of trying radium bromide, and, having no data to guide me in its application, it was tried tentatively at frst, and the following are extracts from my casebook.

On May 2 st, r s $_{3}$, a tube of $5 \mathrm{mg}$. of radium bromide was placed on the upper part of the ulcer for fifteen minutes, and again on May $22 n d$ as before, only further down.

The salt was always shaken down to one end of the tube, so that the point of application was definite. The tube was held in position by a strap of rubber plaster put across the upper part of the tube which fixed it to the skin.

On May 26 th there was 'apparently slight reaction. Two tubes now applied for a short time (exact time not recorded). On May 29 th the ulcer was apparently greatly improved; its edges were levelling. The tubes were applied for twelve minutes. On June $r$ th the ulcer was practically healed, but some thickening could be felt at the upper porder. Two tubes were applied for eighteen minutes, and on June 26 th they were applied for eight minutes over a slightly thickened part.

On July roth a slight tendency to breaking down was noticed in the centre, and one tube applied (time not stated). Treatment was now stopped.

This patient was carefully examined on December 7 th, and there was no trace of recurrence; the skin was quite natural over the part and freely movable, and it was only on very careful inspection that any difference could be detected between the two sides of the nose. Thus for five months there has been no recurrence.

\section{CASE II.-Tuberculosis Verucosa Cutis.}

P. V., aged ro. Sent to me by Dr. Macleod. The patch, about. the size of a sixpence, was situated on the skin of the palm of the right hand, between thumb and forefinger. It had been under treatment for three years.

June isth. Two tubes of radium applied for twenty-five minutes.

June 15 th. Two tubes for twenty-two minutes.

June 28 th. Two tubes for twenty minutes.

July 13 th. Two tubes for thirty-five minutes.

July r6th. Greatly improved; two tubes applied for thirty-five minutes.

July 2oth. Two tubes for half an hour. July 22 nd. Two tubes applied for twenty minutes. The patient went to Dr. Macleod, who tells me that there was a small spot, to which he applied a radium tube, and this effected a complete cure.

\section{CAse III.-Rodent Cancer.}

Miss S., suffering from rodent cancer of the nose, was sent to me is

Mr. Rickman Godlee.

July $22 \mathrm{nd}, 23 \mathrm{rd}, 24 \mathrm{th}, 25^{\mathrm{th}}$. Two tubes applied for thirty minutes. The tubes were placed on different parts of the ulcer on each occasion. There was no reaction.

July 28 th. Greatly improved. Two tubes applied for forty minutes in one position and for twenty minutes in another.

August th A blush round the border, but thickening all igone. Yellow crusts in centre.

August zoth. Greatly improved. The crust came away. Put single ube in the centre for half an hour.

August 3 oth. Put single tube in lower border, where there is a slight thickening left.

September 24 th. Quite cured, apparently. Placed a single tube at. lower part, where there are a few dry scales, for fifteen minutes.

October $x_{5}$ th. Cured.

CASE IV.-Rodent Ulcer or Epithelioma.

Miss H. presented what appears to be an extensive rodent ulcer of ight side of the face.

This case was brought to me by Dr. Powell. It seemed hopeless. Three operations had been performed, recurrence following; $x$ rays had been tried with a slight improvement at first, only to be followed by what appeared to be increased destructive activity. The surgeons: y of epithelioma.

of epithelioma. The condition when I first saw her was as follows: The whole of the right cheek had been destroyed, the tongue and teeth being laid bare; the upper and lower lips were destroyed to within an inch of the middie line; the patient could not speak. The tongue was foul, and there was a very offensive discharge from the

pare provement could be readily noted. If arrest or the process occurred, 